\title{
Errors Associated with Aggregating Asian American Ethnic Groups in Diabetes Research, Education, and Clinical Practice
}

\author{
Deovina N. Jordan ${ }^{1 *}$, James L. Jordan², Neil John Yumul ${ }^{3}$, Janine Marie Baptista ${ }^{4}$ \\ ${ }^{1}$ Mattel Children's Hospital, 3F Ronald Reagan UCLA Medical Center 757 Westwood Boulevard Los Angeles, \\ California, USA \\ ${ }^{2}$ Dr. Jordan and Dr. Jordan Murrieta, California, USA \\ ${ }^{3}$ Linda Valley Care Center Loma Linda, California, USA \\ ${ }^{4}$ Las Pinas General Hospital and Satellite Trauma Center Las Pinas City, Metro Manila, Philippines
}

*Corresponding Author: Deovina N. Jordan, Mattel Children's Hospital, 3F Ronald Reagan UCLA Medical Center 757 Westwood Boulevard Los Aneeles. California. USA. Email: diordan@mednet.ucla.edu

\begin{abstract}
Asian American ethnic groups are often aggregated into the classification of Asian American. To aggregate Asian American ethnic groups, certain assumptions about their homogeneity must be made. These assumptions may include the following: First, physical characteristics do not vary among Asian American ethnic groups. Second, cultural and dietary factors contributing to health in Asian American ethnic groups must be identical or have very little variation. Third, Asian and Asian American ethnic groups must be genetically homogenous. Fourth, the risk and/or prevalence for type 2 diabetes mellitus (T2DM) must be the same across Asian American ethnic groups. However, different Asian American ethnic groups cannot be aggregated based on those assumptions. The ramifications of the aggregation of Asian American ethnic groups and extrapolation of data from one Asian American ethnic group to another have important and serious adverse consequences in terms of research, educational efforts and clinical interventions for Asian Americans.
\end{abstract}

Keywords: Asian Indian, Chinese, Clinical Practice, Complications, Extrapolation, Filipino, Japanese, Korean, Vietnamese, Research

Abbreviation: T2DM: type 2 diabetes mellitus, LDL: low density lipoprotein, BMI: body mass index.

\section{INTRODUCTION}

\subsection{Aggregating Asian American Ethnic Groups into a Single Ethnic Group}

A common practice in the medical literature, both clinical and research oriented, is to aggregate ethnic groups from Asia into the categories of "Asian" or, if living in the United States, "Asian American." In fact, most of the information regarding prevalence of T2DM among Asian Americans is aggregated across Asian American ethnic groups [1].Consequently, specific information about the incidence and prevalence of T2DM among the Asian Americans remains scanty [2].

The term Asian is associated with at least 32 distinct subgroups that have their origins in any of the original people of the Far East, the Indian subcontinent, or Southeast Asia [3]. As such, a consequence of aggregating data is that a wide variety of combinations of Asian American ethnic groups can occur in both the classification of "Asian" and "Asian American."

The resultant aggregation of Asian American ethnic groups (as per published articles) into Asian American can include these Asian ethnic groups living in the United States: South Asian [2], Sri Lankan [4], Pakistani [4], Burmese [4], Cambodian [4],Malaysian [4], multiracial [2, 4], Chinese [2, 4-10], Asian Indian [4-6], Korean [4-6, 8, 9], Vietnamese [4, 6-8, 10], Filipino [4, 6-10], Japanese [4, 7, 8], other [8], and/or Hmong [10]. Sometimes, aggregation is expanded to include members of the Pacific Islander ethnic groups [11]. As such, the sample composition used in different studies and reports varies considerably in terms of the composition of Asian American ethnic groups. Statistically speaking, the samples used in aggregated studies are not representative of the composition of the Asian American community as a whole. This is compounded by the use of self report data $[7,8]$ or very small sample size 
(e.g., 12 persons of Asian ethnicity) [12]. For example, some studies utilized data collected via a statewide survey $[7,8]$. As such, no confirmation was obtained on their specific ethnicity or health problems. The consequences of utilizing diverse Asian American compositions in research studies, which are not representative of the Asian American population as a whole, will be discussed later in this article.

Authors and researchers of some studies [4, 10, 13] reported that their research population was overwhelmingly one Asian ethnic group. For example, a study by Hung et al.[4]utilized $77 \%$ Chinese subjects while a study by Wu et al. [10] utilized 67\% Chinese participants. Both studies claimed that the Chinese ethnic group is representative of Asian Americans. A consequence of differential treatment of Chinese participants from those of other Asian American ethnic groups was a bias in using Chinese Americans as representative of Asian Americans as a whole.

The bias towards a specific Asian American ethnic group can arise due to the selection of specific translators or the use of instruments translated for specific Asian American ethnic groups only. For example, Wong, Chou, Sinha, et al.[13] reported a study in which bilingual interviewers administered surveys only in Spanish, Mandarin, and Cantonese.

Selection bias is not oriented solely toward having Chinese Americans as representative of Asian Americans as a whole. One report discussed Asian Americans and T2DM but used Japanese Americans exclusively [14]. Another study applied the results to all Asian Americans even when sufficient sample size was available to perform statistical analysis only for Asian Indians, Chinese, and Koreans [5]. Sufficient sample size in research studies may be present for each Asian American ethnic group, but even then some Asian American ethnic groups may be represented more than other Asian American ethnic groups. On top of that, even gender can be skewed. For example, Patterson, et al. [15] reported a research study involving $82 \%$ female in a sample consisting of $45 \%$ Vietnamese, $38 \%$ Chinese, and 17\% Korean. Moreover, even in cases when the research sample is predominantly one to three Asian American ethnic groups, the findings are applied to all Asian Americans. As such, if a sample of hundreds to thousands of Asian Americans was predominantly one ethnic group, with only few participants from other Asian American ethnic groups, the results of the thousands (the predominant Asian American group) were reported as being representative for all members of the Asian American group represented by very few individuals $[4,10,15]$. The incorrect extrapolation of data from one Asian American ethnicity was commented on by Holland and Palaniappan [16]. They noted that findings of the Ni-Hon-San study about Japanese and Japanese Americans had been incorrectly extrapolated to other Asian American ethnic groups.

Aggregation of Asian American ethnic groups can often ignore the actual composition of the Asian American population. The six largest Asian American ethnic groups (Asian Indians, Chinese, Filipinos, Japanese, Koreans, and Vietnamese) comprise between $84 \%$ [17] to 97\% [16] of the Asian American population. As such, the use of one, two or more of these Asian American ethnic groups, though having the largest number in terms of population, may not even reflect the actual Asian American composition. Some consequences of having one Asian American ethnic group as dominant, but extrapolating the results throughout the Asian American population, are discussed later in this article.

In some cases, the term Asian American can simply mean that a subject in the study was simply born in an Asian country [18]. Finally, the terms Asian and Asian American may not even address the Asian or Asian American ethnic groups included; the reader is simply expected to know and/or accept [11, 12, 19-26]. Not knowing the composition of an aggregated sample has notable consequences in terms of statistical analysis, replication of the study, current and future research, and evidence-based clinical practice.

\section{DISCUSSION}

\subsection{Possible Assumptions made to Aggregate Asian American Ethnic Groups}

Aggregation of data depends upon some homogeneity among the groups being aggregated. As such, assumptions leading to aggregation of Asian American ethnic groups into the designation of Asian American may include: (1) physical characteristics do not vary among Asian American ethnic groups; (2) 
Errors Associated with Aggregating Asian American Ethnic Groups in Diabetes Research, Education, and Clinical Practice

cultural and dietary factors contributing to health in Asian American ethnic groups must be identical or have very little variation; (3) Asian and Asian American ethnic groups must be genetically homogenous; and (4) the risk and/or prevalence for T2DM must be the same across Asian American ethnic groups. The assumptions that may be necessary to aggregate Asian American ethnic groups into the category of Asian American will be discussed. After the discussion, consequences of faulty aggregation of Asian Americans will be addressed.

\subsection{Assumption: Physical Characteristics do not Vary among Asian American Ethnic Groups}

Comparisons of being overweight or obese are objective measures of determining if physical characteristics are comparable across Asian American ethnic groups. According to Holland et al.[27], the percentages of overweight participants in their study were: 45.1 for Koreans, 39.7 for Asian Indians, 39.7 for Filipinos, 33.4 for Japanese, 33.1 for Chinese, and 28 for Vietnamese. The percentages of obese participants in their study were: 24.9 for Filipinos, 22.4 for Japanese, 20.5 for Asian Indians, 12.9 for Chinese, 11.3 for Koreans, and 9.3 for Vietnamese. The body mass index (BMI) may prove to be a reliable indicator of cardiometabolic risk. However, in a study involving Chinese American and South Asian American participants, Gujral et al.[28, pg. 628] reported that "Using a BMI criterion for overweight to screen for cardiometabolic risk may result in a large proportion of racial/ethnic minority groups being overlooked."That can be problematic when aggregation can lead to the underestimation of risks and prevalences.

Another objective measure of physical health is the level of low density lipoprotein (LDL). Holland et al.[27] reported the mean LDLs for the following populations: Vietnamese (101.2), Filipinos (98.3), Asian Indians (98.1), Koreans (97.4), Chinese (95.4), and Japanese (95.1). Based on the physical characteristics of being overweight or obese and levels of LDL, one can conclude that physical characteristics are not the same across Asian American ethnic subgroups. Because of the varying physical characteristics among Asian American ethnic groups, the different Asian American ethnic subgroups should not be aggregated into an overall classification of Asian American.

\subsection{Assumption: Cultural and Dietary Factors Contributing to Health in Asian American Ethnic Groups must be Identical or have Very Little Variation}

Aggregating Asian American ethnic groups essentially treats Asian American ethnic groups as a homogeneous group. That homogeneity would have to arise from having origins from Asia and the myth of being a model minority [23]. But, as Nguyen et al.[23] indicated, Asian Americans are heterogenous and differ regarding self-sufficiency, education, and burdens of disease (particularly regarding risk for and prevalence of diabetes). Islam, TrinhShevrin, and Rey, [29] also emphasized the racial and ethnic diversity of Asian Americans having distinct cultures, health behaviors/ practices (e.g., exercise), and lifestyle habits (e.g., diets). The authors concluded that aggregation of Asian American ethnic groups obstructed identification of those at high risk for T2DM, thereby impacting prevention and intervention strategies [29].

The fact that Asian American ethnic groups are not homogenous, regarding cultural aspects impacting health, was also addressed by $\mathrm{Wu}$ et al.[10, page 811] when they wrote: "Attention needs to be paid to the extent and nature of ethnic-specific health problems because each culture's health-related habits and beliefs affect their entry into preventive care so that strategies can be designed and implemented to effectively reduce and ultimately eliminate health disparities."

One health related behavior is smoking. If the Asian American ethnic groups have the same health related behaviors, smoking should be uniform across the different groups. However, according to Holland et al.[27], the last available smoking status in their study was $33.1 \%$ for Koreans, $30.1 \%$ for Filipinos, $28.4 \%$ for Japanese, $16.8 \%$ for Vietnamese, $16.7 \%$ for Asian Indians, and $16.7 \%$ for Chinese. On the basis of smoking alone, Asian Americans from different ethnic groups do not practice the same health related behaviors.

Another health related behavior is diet. Asian American ethnic groups have different diets. About a third of South Asians practice vegetarianism [30]. In contrast, Filipino Americans have diets high in animal protein [31]. In addition, Filipino American diets are high in salt [32]. Likewise, Korean Americans have diets that are high in salt [33]. 
Acculturation (the adoption of a more Westernized lifestyle) compounds the issue of diet within Asian American ethnic groups. For example, consumption of meat, fruits and fresh vegetables increased, milk included, and starchy foods and snacks decreased as Filipino Americans became increasingly acculturated [34]. The dietary addition of milk, cheese, and fatty foods; increased fruit and vegetable intake; fast food restaurant use; and eating between meals were reported as being components of Chinese Americans becoming acculturated [35, 36]. Consequently, acculturation, since it impacts diet, increases the variability of health both within and among Asian American ethnic groups. The causes behind acculturation have not been truly explored in scientific research. It could be due to the adoption of components of the predominant new culture or it could be due to increased economic resources available to the immigrant population; thereby, increasing the population's ability to purchase foods that had been previously unaffordable and/or unavailable.

Health related behaviors can be influenced by the different religions practiced by different Asian American ethnic groups from different regions of Asia. Atheism is common among Chinese (the official stance of Communist China) and Vietnamese (81.8\%) [37]. Buddhism is embraced by most Thais (93.6\%), Taiwanese (with Taoism) (93\%), Bhutanese (75.3\%), Sri Lankans (70.2\%), Japanese (66.8\%), along with sizeable Korean (24.2\%), Malaysian (19.8\%), and Chinese $(18.1 \%)$ minorities. Christianity is the religion of most Filipinos $(82.9 \%$ Roman Catholic, 9.6\% other Christian) along with sizeable South Korean (31.6\%), Indonesian (9.9\%), Malaysian $(9.2 \%)$, and Chinese $(5.1 \%)$ minorities. Hinduism is the religion of many Asian Indians (79.8\%) and sizeable Sri Lankan $(12.6 \%)$ and Malaysian (6.3\%) minorities. Islam is practiced extensively among Pakistani (96.4\%), Bangladeshi (88.1\%), Indonesian (87.2\%), Malaysian (61.3\%), and Asian Indian (14.2\%) populations. Shintoism is practiced by Japanese (79.2\%) [37]. As such, Asian Americans develop different health practices associated with the religions of choice from their lands of origin. Such practices include fasting; fish, beef and/or pork consumption; vegetarianism; and rice intake. Aggregation of Asian American ethnic groups also hides the importance that religion plays in the different diets, health risks and behaviors. As such, aggregation becomes culturally insensitive in that it treats all Asian American ethnic groups as being the same, even when clearly distinct based on their religious practices alone. These differences not only impact the risks and prevalence of diseases, but also the appropriate preventive, educational and therapeutic measures necessary.

Finally, health related behaviors, including diet, are influenced by the environment in which a population resides. Asia has tropical forests, temperate forests, deserts, grasslands, coasts, mountains, and plains. People adapt accordingly and differently. When they translocate, such as immigrating to the United States, they may either acculturate or maintain some of their environmentally affected behaviors even though they live in a totally new environment. For example, someone from a tropical region in Asia may still seek to eat the same foods even when living in the northern United States. Immigration also affects the incomes of many individuals. After immigrating and getting better paying jobs, they may be able to purchase foods that had been cost-prohibitive in their places of origin. Aggregating across Asian American ethnic groups has the de-facto assumption that the origins of those groups are similar environments with comparable behavioral adaptations and economic opportunities.

The assumption that cultural and dietary factors contributing to the health of Asian Americans are identical (or have very little variation) is not substantiated. When data regarding food consumption is based upon aggregated data for Asian Americans [38], applying the same data to specific Asian American ethnic groups is not plausible since the data has no meaningful significance to specific Asian American ethnic groups. Doing so may lead to culturally inaccurate educational and clinical interventions to either reduce incidence of or properly treat T2DM among Asian American ethnic groups. Therefore, the assumption mentioned above cannot be used as a basis for aggregation of Asian American ethnic groups.

\subsection{Assumption: Asian and Asian American Ethnic Groups must be Genetically Homogenous}

Out of 130 ancestry informative singlenucleotide polymorphism (AISNP) panels investigated in one study, 122 were found to be useful for differentiating among five Asian 
Errors Associated with Aggregating Asian American Ethnic Groups in Diabetes Research, Education, and Clinical Practice

populations, namely Filipinos, Indonesians, Taiwanese Han Chinese, Thais, and Vietnamese [39]. Genetic research has also shown the unique genetic characteristics of Han Chinese (80 genome copy number variation regions) [40], Japanese (as Hondo, Ryuku, and Ainu) [41], northern Thai [42], and Tibetans [43].

Asian ethnic groups, once considered to be large homogenous groups, are actually composed of many genetically heterogenous groups. For example, South Asians, from 260 South Asian groups, were clustered into 81 genetically distinct groups [44]. For Asian Indians, the number of genetically distinct groups may be higher than that. Liu et al.[45] investigated 140 distinct genomic regions of 37 cosmopolitan and ethnic minority populations in Asia. They noted that minority populations, particularly in South Asia, had different adaptations when compared to populations from other geographical regions [45]. Some ethnic diversity in South Asia is linked to the Y-chromosome. Mondal et al.[46] reported 42 new Y- chromosomal sequences unique to different South Asian tribal and nontribal populations. If Asian or Asian American ethnic groups are to be aggregated, genetic information already indicates that South Asians and South Asian Americans should not be included with non South Asian and non South Asian American ethnic groups (respectively). Aggregation of South Asians and South Asian Americans already provides a wide diversity of genetically different ethnic groups.

To use the assumption that Asian and Asian American ethnic groups are genetically homogenous and therefore aggregatable should follow the logic that specific disorders are not linked to specific Asian or Asian American ethnic groups. If specific disorders can be linked specifically to a particular Asian or Asian American ethnic group, then it would follow that the groups are not homogeneous and therefore should not be aggregated. An example of heterogeneity among Asian American ethnic groups can be seen with cardiovascular risks. Wu et al.[10] reported differences among Asian American ethnic groups regarding cardiovascular risks. They reported that Chinese and Filipino participants in their study were at risk for cardiovascular disease notably due to hypertension and cholesterol problems. In contrast, Hmong participants in their study were placed at risk for cardiovascular disease largely due to having a higher risk of diabetes when compared to Chinese, Filipino, and Vietnamese participants [10]. As such, even on the basis of cardiovascular risk factors alone, Asian or Asian American ethnic groups should not be simply aggregated.

\subsection{Assumption: Risk and/or Prevalence for T2DM must be the Same Across Asian American Ethnic Groups}

The Centers for Disease Control and Prevention [47] reported that the age-adjusted risk for T2DM is moderate (9\%) for Asian Americans. However, the prevalence of T2DM varies considerably among different Asian American ethnic groups as a consequence of heterogeneity [48]. When aggregated data for Asian Americans was disaggregated, the age-adjusted prevalences for T2DM were: $15.8 \%$ for Filipino males and $9.4 \%$ for Filipino females; $11.8 \%$ for Japanese males and $7.6 \%$ for Japanese females; $6.7 \%$ for Korean males and $5.1 \%$ for Korean females; $6.3 \%$ for South Asian males and $2.7 \%$ for South Asian females; $5.0 \%$ for Chinese males and 3.6\% for Chinese females; and $2.5 \%$ for Vietnamese males and $2.1 \%$ for Vietnamese females [23, 49].Wang et al.[48] also reported statistically significant differences in the prevalence of T2DM for different Asian American ethnic groups. In their study, the prevalence was $14.8 \%$ for Filipinos, $11.2 \%$ for Asian Indians, $8.3 \%$ for Japanese, $5.5 \%$ for Chinese, $4.3 \%$ for Koreans, and $4.3 \%$ for Vietnamese. Another study, conducted by Karter et al.[2] contained different estimates of T2DM among various Asian American ethnic groups. They reported the prevalence as follows: $16.13 \%$ for Filipinos, $15.85 \%$ for South Indians, $10.28 \%$ for Japanese, $9.85 \%$ for Koreans, $9.85 \%$ for Vietnamese, and $8.15 \%$ for Chinese. Finally, the prevalence of T2DM can be even higher among segments of different Asian American ethnic groups. In a study conducted in Hawaii, Sentill et al.[9] reported prevalences (both diagnosed and potentially undiagnosed, respectively) as: $34 \%$ and $3 \%$ for Japanese Americans; $33 \%$ and $4 \%$ for Filipino Americans; and $32 \%$ and $4 \%$ for Chinese Americans.

Karter et al. [2, page 577] reported a major problem in the aggregating of Asian Americans regarding diabetes when they wrote about "the risk of the larger groups(Chinese and Filipinos) exerting a stronger influence on the overall rate. 
Errors Associated with Aggregating Asian American Ethnic Groups in Diabetes Research, Education, and Clinical Practice

The very high risk experienced among Pacific Islanders, South Asians, and Filipinos is masked in the aggregate rate by the much lower risk among Chinese and Japanese." According to Nguyen et al.[1], the disaggregated data of Asian Americans showed that prevalence of diabetes was highest for Filipino, Japanese, and South Asian ethnic groups. They also pointed out that aggregating data on Asian American ethnic groups can mask the risk of T2DM for those at high risk, while inflating the risk for those at low risk [1].

An important component of the studies reporting diabetes prevalence for different Asian American ethnic groups is that they usually differ considerably among the different Asian American ethnic groups. Consequently, information regarding Vietnamese or Vietnamese Americans does not necessary apply to Filipinos or Filipino Americans. Therefore, aggregation of Asian American ethnic groups into a single classification for the study of T2DM is feasible only if the ethnic groups are somewhat homogenous.

\section{Consequences of AgGRegating}

\subsection{Complication Risks/ Prevalences of T2DM and other Diseases are Underestimated or Overestimated}

Aggregation of Asian American ethnic groups into one group, namely Asian American, does not only underestimate the prevalence of T2DM among some Asian American groups but also overestimate the prevalence in other Asian American groups. The underestimation and overestimation of the prevalence of T2DM also leads to the underestimation and overestimation of complications associated with T2DM. For some groups, proper estimation of the prevalence of T2DM affects mortality. Diabetes by itself accounts for $5 \%$ of all deaths of both Asian Indian and Filipino women [50]. But, the complications of diabetes can also be lethal. Since the focus of this article is on aggregation of Asian American ethnic groups, the complications of T2DM cannot be fully discussed in this paper.

A notable complication of T2DM is heart disease [33]. About three-fourths of deaths in persons with T2DM are a consequence of cardiovascular diseases [51, 52]. The relative frequency of heart disease as a cause of death varies among Asian American ethnic groups. Death from heart disease accounts for $23 \%$ of Japanese American, 22\% of Korean American, and $19 \%$ of Vietnamese American deaths overall [50]. When gender is factored in, heart disease is the leading cause of death for $28.2 \%$ of Filipino males, 27.2\% of Japanese males, and $18 \%$ of Asian Indian males. Heart disease is the second leading cause of death for $23.2 \%$ of Chinese males, $19.2 \%$ of Korean males, and $17.8 \%$ of Vietnamese males. Heart disease is the leading cause of death for $27.8 \%$ of Asian Indian females, but the second leading cause of death for $24.4 \%$ of Filipino females, $23.7 \%$ of Chinese females, $22.6 \%$ of Japanese females, $21.6 \%$ of Korean females, and $19.1 \%$ of Vietnamese females [50].

Another complication of T2DM is anemia [53]. Anemia, as a complication of T2DM, has been associated with an increased risk for hypoxiainduced organ (including cardiovascular) damage and death [53].

The prevalence of life-threatening complications of T2DM for Asian American ethnic groups can be underestimated or overestimated when Asian American ethnic groups are aggregated. As Hastings et al. [50] noted: "The aggregation of Asian Americans masks important differences in mortality among subgroups."

\subsection{Impact of Aggregating Asian American Ethnic Groups on Research}

Aggregating Asian American ethnic groups can have major consequences on research and upon the usefulness of the research study results. If the Asian American ethnic groups being aggregated are primarily from one Asian American ethnic group, then the variance of the data can be smaller than when several Asian American ethnic groups have been aggregated. A consequence of less variance is that comparison of the findings with non Asian American ethnic groups can be more often statistically significant. The finding that statistical significance is present, but wrong, is called a type 1 error. In contrast, if the variance is greater, the results may indicate that no statistically significant difference exists between Asian Americans and another ethnic/racial group (e.g., non Hispanic Whites, Hispanics, or Blacks). The finding that no statistically significant difference exists between groups when a statistically significant difference is actually present is called a type 2 error. It is correctable. Adequate sample sizes from each Asian American ethnic group are necessary for research studies rather than simply aggregating 
Errors Associated with Aggregating Asian American Ethnic Groups in Diabetes Research, Education, and Clinical Practice

data from different Asian American ethnic groups.

The differences in significance can directly impact patient care (especially evidence-based patient care). For example, while studies may indicate that the application of glycated hemoglobin (HbA1C) may be appropriate to diagnose or measure glycemic control of T2DM [24] in Chinese Americans [54], that conclusion would be lead to problems when applied to Filipino Americans. Araneta et al.[55] noted that Filipino Americans have a low sensitivity to the application of glycated hemoglobin (HbA1C) in the diagnosis T2DM. Thus, if glycated hemoglobin (HbA1C) was to be used to diagnose T2DM for Filipino Americans, the diagnosis of T2DM in this population would be delayed along with requisite preventive or treatment interventions.

Aggregating Asian American ethnic groups also reduces the reproducibility of studies. The reason is that the results and the conclusions of the studies are dependent upon the selection of certain Asian American ethnic groups to represent Asian Americans in general. If studies cannot be replicated, then it becomes more difficult to derive conclusions as to which studies are most valid. Moreover, when study samples are not representative of the overall population for which the results may be applied to, generalizing the results from the sample to the overall population is no longer feasible due to the findings being erroneous. So, while the results of aggregated studies may be statistically significant, they may actually be no longer meaningful or clinically applicable/relevant.

Aggregating Asian American ethnic groups also impacts the focus and performance of future research and research funding. Research tends to go in the direction that yields publishable results and research funding is founded upon studies supported by previous research. As a consequence, if previous research studies have errors, those errors are likely to be reproduced in future research.

\subsection{Impact of Aggregating Asian American Ethnic Groups on Evidence-Based Clinical Practice}

Aggregating Asian American ethnic groups in research studies has more than a direct impact on evidence-based clinical practice. Aggregating Asian American ethnic groups into a single classification impacts translational research which seeks to interpret research findings to be applicable to the clinical setting. For example, knowing the health of particular Asian American ethnic groups becomes difficult. As Abesamis et al.[32] noted, regarding Filipino American health, "The general status and overall picture ... is difficult to discern from existing literature because national health surveys generally combine individuals of different Asian groups together." That is unfortunate. Aggregating Asian American ethnic groups then has the consequence of not helping, but harming, the Asian Americans who were intended to be helped by the research studies. This is particularly true when current clinical practice translates and incorporates scientific research into clinical practice (evidence-based practice) such as in the pharmaceutical industry. Data from one Asian American ethnic group has been incorrectly extrapolated throughout the Asian American ethnic groups, thereby disregarding critical genetic differences [27].

Evidence-based practice has its foundation on the concept that research evidence should provide the basis for clinical practice. But, that foundation quickly erodes when the research evidence is actually faulty, such as when the aggregating of groups leads to the overestimation or underestimation of risks for specific groups. By overestimating the risk, some groups may be over screened or over treated. Over screening and/or over treating are not economically sound since limited resources have to be used on the assumption that the risk is greater than it actually is. By underestimating the risk, some groups may be inadequately screened and may not receive sufficient treatment. This is also economically unsound since the overstretched resources may not be appropriately used as they may be applied only after complications [that need more expensive interventions] have developed. For example, undiagnosed $\mathrm{T} 2 \mathrm{DM}$ is associated with a significant increase in hospitalizations [9]. In addition, prevention and education efforts may be influenced by perceptions of risk which may be inaccurate due to aggregation.

\section{CONCLUSION/RECOMMENDATIONS}

Aggregating Asian American ethnic groups into the single category of Asian American is not supported by common physical characteristics, similar cultural and dietary factors, genetic homogeneity, or uniform risk/prevalence for 
T2DM among Asian Americans. In order to prevent underestimation or overestimation of disease (e.g., T2DM) risk and prevalence, along with associated complications, data should not be extrapolated from one Asian American ethnic group to another. A component of the solution is to have sufficient sample size for each Asian American ethnic group in research studies, thereby ensuring adequate power for better statistical results. Moreover, in order to replicate research studies, the Asian American ethnic groups in studies have to be identified. Not identifying Asian American ethnic groups in research studies prevents the replication of such studies (an important component of good research). Additionally, not identifying Asian American ethnic group samples diminishes the research, educational, and clinical applicability of research studies. Finally, since future research and current clinical practices are adversely impacted by the aggregation of Asian American ethnic groups and extrapolation of data from one Asian American ethnic group to another, aggregating dissimilar groups is strongly discouraged.

\section{REFERENCES}

[1] Nguyen T. H., Nguyen T., Fischer T., Ha W.and Tran V., Type 2 diabetes among Asian Americans: prevalence and prevention, World J. Diabetes. 6(4), 543-547 (2015).

[2] Karter A. J., Liu J., Schillinger D., Adler N. E., Adams A, S, Kanaya A. M. and Moffet, H. H., Elevated rates of diabetes in Pacific Islanders and Asian subgroups; the Diabetes Study of Northern California (DISTANCE), Diabetes Care.36, 574-579 (2013).

[3] Office of Management and Budget, The 1997 Revisions tothe Standards for the Classification of Federal Data on Raceand Ethnicity, Available from: URL: https://www. whitehouse. gov/omb/fedreg_1997 standards (2015).

[4] Hung L., Wong S., Iftakar T., Keenan H., King L. and Hsu, W. C., Characterization of factors affecting attainment of glycemic control in Asian Americans with diabetes in a culturally specific program, Diabetes Educ. 39(4), 468477 (2013).

[5] Islam N. S., Kwon S. C., Wyatt L. C., Ruddock C., Horowitz C. R., Devia C. and TrinhShevrin,C., Disparities in diabetes management in Asian Americans in New York City compared to other racial/ethnic minority groups, American J. Public Health. 105(53), S443-S446 (2015).

[6] Staimez L. R., Weber M. B., Narayan K. M. V. andOza-Frank, R., A systematic review of overweight, obesity, and type 2 diabetes among Asian American subgroups. Curr. Diabetes Rev. 9(4), 312-331 (2013).

[7] Becerra M. B. and Becerra B. J., Disparities in age at diabetes diagnosis among Asian Americans: implications for early preventive measures, Prev. Chronic Dis. 12,E146 (2015).

[8] Mui P., Bowie J. V., Juon H. and Thorpe, R. J., Ethnic group differences in health outcomes among Asian American men in California, Am. J. Men's Health. 11(5), 1406-1414 (2016).

[9] Sentill T. L., Cheng Y., Saito E., Seto T. B., Miyamura J. et al., The burden of diagnosed and undiagnosed diabetes in native Hawaiian and Asian American hospitalized patients, J. Clin. Transl. Endocrinol. 2(4), 115-124 (2015).

[10] Wu T., Hsing-Fang H., Wang J., Yao L. and Oakley D., Ethnicity and cardiovascular risk factors among Asian Americans residing in Michigan, J. Community Health. 36, 811-818 (2011).

[11] Fukuoka Y., Lisha N. E. and VittinghoffE., Comparing Asian American women's knowledge, self-efficacy, and perceived risk of heart attack to other racial and ethnic groups: the mPED trial, J. Women's Health.26(9), 1012-1019 (2017).

[12] Huang J. P., Roundtree A. K., Engebretson J. C. and Suarez-Almazor M. E., Medical care of hepatitis B among Asian American populations: perspectives from three provider groups, J. Gen. Intern. Med.25(3), 220-227 (2009).

[13] Wong R. J., Chou C., Sinha S. R., Kamal A. and Ahmed A., Ethnic disparities in the association of body mass index with the risk of hypertension and diabetes, J. Community Health.39, 437-445 (2013).

[14] Royal Society for Public Health. Waist measurement as an aid to type II diabetes screening among Asian Americans, Perspectives in Public Health. 137(1), 36-37 (2016).

[15] Patterson F., Zhang G., Davey A., Tan Y. and Ma G. X., American Heart Association's ideal cardiovascular health metrics in underrepresented Asian Americans, J. Community Health. 41(6), 1282-1289 (2016).

[16] Holland A. T. and Palaniappan L. P., Problems with the collection and interpretation of AsianAmerican health data: omission, aggregation, and extrapolation, Ann. Epidemiol. 22(6), 397405 (2012).

[17] United States Census Bureau, Projections of the Population by Sex, Race, and Hispanic Origin for theUnited States: 2015 to 2060. Washington D.C., U.S. Census Bureau (2012). 
[18] Pollack H. J., Kwon S. C., Wang S. H., Wyatt L. C. and Trinh-Shevrin C., Chronic hepatitis B and liver cancer risks among Asian-immigrants in New York City: results from a largecommunity based screening, evaluation, and treatment program, Cancer Epidemiol. Biomarkers. Prev. 23(11), 2229-2239 (2014).

[19] Khalili M., Lombardero M., Chung R. T., Terrault N. A., Ghany W. et al., Diabetes and prediabetes in patients with hepatitis $\mathrm{B}$ residing in North America, Hepatology. 62(5), 13641374 (2015).

[20] Koshizaka M., Lopes R. D., Newby L. K., Clare R. M., Schulte P. J. Tricoci P. et al.,Obesity, diabetes, and acute coronary syndrome: differences between Asians and Whites,Am. J. Med. 130, 1170-1176 (2017).

[21] Lopez J. M. S., Bailey R. A., Rupnow M. F. T. and Annunziata, K., Characterization of type 2 diabetes mellitus burden by age and ethnic groups based on a nationwide survey, Clinical Therapeutics.36(4), 494-506 (2014).

[22] Tung E. L., Baig A. A., Huang E. S., Laitterapong N. and Chua K., Racial and ethnic disparities in diabetes screening between Asian Americans and other adults: BRFSS 2012-2014, J. Gen. Intern. Med. 32(4), 423429 (2016).

[23] Nguyen A. B., Breen N., Clark T. T. and Moser R., The biracial Asian population of California: an examination of health profiles and chronic conditions, Eth. Dis. 24(4), 481-487 (2014).

[24] Woffenbuttel B. H. R., Dharmalingam M., Herman W. H., Jiang H. H., Gross J. L. and Hardin D. S., Ethnic differences in glycemic markers in patients with type 2 diabetes, Diabetes Care.36, 2931-2936 (2013).

[25] Yoo G. J., Fang T., Zola J. and Dariotis W. M., Destigmatizing hepatitis $\mathrm{B}$ in the Asian American community: lessons learned from the San Francisco Hep B Free Campaign, J.Cancer Educ.27(1), 138-144 (2012).

[26] Zhou Q. and Oh K. M., Comparison of lifestyle behaviors and related factors between Asian American and White adults with prediabetes, Nursing and Health Sciences. 14,58-66 (2012).

[27] Holland A. T., Zhao B., Wong E. C., Choi S. E., Wong N. D. and Palaniappan, L. P.,Racial/ethnic differences in control of cardiovascular risk factors among type 2 diabetes patients in an insured, ambulatory care population, J. Diabetes Complications. 27(1), 33-40(2013).

[28] Gujral U. P., Vittinghoff E., Mongraw-Chaffin M., Vaidya D., Kandula N. R. et al., Cardiometabolic abnormalities among normalweight persons from five racial/ethnic groups in the United States: a cross-sectional analysis of two cohort studies, Ann. Intern.Med.166, 628636 (2017)

[29] Islam N. S., Trinh-Shevrin C. and Rey M. J., Toward a Contextual Understanding of Asian American Healthin Asian American Communities and Health: Context, Research, Policy and Action. San Francisco, CA: John Wiley \& Sons, Inc., 2009,pp. 3-22.

[30] Jaacks L. M., Kapoor D., Singh K., Narayan K. M. V., Ali M. K., Kadir M. M. et al.,Vegetarianism and cardiometabolic disease risk factors: differences between south Asian and American adults, Nutrition. 32(9), 975-984 (2016).

[31] Kim H., Park S., Grandinetti A., Holck P. S. and Waslien, C.,Major dietary patterns, ethnicity, and prevalence of type 2 diabetes in rural Hawaii, Nutrition. 24, 1065-1072(2008).

[32] Abesamis C. J., Fruh S., Hall H., Lemley T. andZlomke K. R., Cardiovascular health of Filipinos in the United States: a review of the literature, J. Transcult. Nurs. 27(5), 518-528 (2016).

[33] Shin C., Keller C., An K. and Sim J., Cardiovascular disease in Korean Americans: a systematic review, J. Cardiovascular Nursing. 00 (0), 00-00 (2017).

[34] Vargas P.and Jurado L., Dietary acculturation among Filipino Americans, Int. J.Environ. Res. Public Health. 13(16) (2015).

[35] Lv N. and Cason K., Dietary pattern change and acculturation of Chinese Americans in Pennsylvania ,J. Am. Dietetic Assoc. 104, 771778 (2004).

[36] Satia J. A., Patterson R. E., Kristal A. R., Hislop T. G., Yasui Y.and Taylor V. M. Development of scales to measure dietary acculturation among Chinese-Americans and Chinese-Canadians, J. Am. Dietetic Assoc. 101(5),548-553 (2001).

[37] Central Intelligence Agency (CIA), The CIA World Factbook 2017, Skyhorse Publishing, New York, New York, 2017.

[38] Sorkin D. H. and Billimek J.,Dietary behaviors of a racially and ethnically diversesample of overweight and obese Californians, Health Education \& Behavior. 39(6), 737-744 (2012).

[39] Hwa H., Lin C., Huang T., Kuo P., Hsieh W. et al., A panel of 130 autosomal single-nucleotide polymorphisms for ancestry assignment in five Asian populations and in Caucasians, Forensic Sci. Med. Pathol. 13, 177-187 (2017).

[40] Lu J., Lou H., Fu R., Lu D., Zhang F. et al., Assessing genome-wide copy number variation in the Han Chinese population, J. Med. Genet. 54, 685-692 (2017). 
Errors Associated with Aggregating Asian American Ethnic Groups in Diabetes Research, Education, and Clinical Practice

[41] Takeuchi F., Katsuya T., Kimura R., Nabika T., Isomura M., Ohkubo T. et al., The fine-scale genetic structure and evolution of the Japanese population,PLoS One, 12(11),e0185487 (2017).

[42] Brunelli A., Kampuansai J., Selelstad M., Lomthaisong K., Kangwanpong D. et al., Y chromosomal evidence on the origin of northern Thai people, PLoS One, 12(7), e0181935 (2017).

[43] Jeong C., Peter B. M., Basnyat B., Neupane M., Beall C. et al., A longitudinal cline characterizes the genetic structure of human populations in the Tibetan plateau, PLoSOne, 12(4), e0175885 (2017).

[44] Nakatsuka N., Moorjani P., Rai N., Sarkar B., Tandon A., Patterson N. et al., The promise of discovering population-specific diseaseassociated genes in South Asia, Nature Genetics. 149(9), 1403-1407 (2017).

[45] Liu X., Lu D., Saw W., Shaw P. J., Wangkumhang P. et al., Characterising private and shared signatures of positive selection in 37 Asian populations, European J. Human Genetics. 25, 499-508 (2017).

[46] Mondal M., Bergström A., Xue Y., Calafell F., Laayouni $\mathrm{H}$. et al., Y-chromosomal sequences of diverse Indian populations and the ancestry of the Andamanese, Hum. Genet. 136, 499-510 (2017).

[47] Centers for Disease Control and Prevention, National Diabetes Statistics Report: Estimates of Diabetes and Its Burden in the UnitedStates, Atlanta, GA: U.S. Department of Health and Human Services, 2014, Available from: URL:http://www.cdc.gov/diabetes/pubs/statsre port14/national-diabetes-report-web.pdf.

[48] Wang E. J., Wong E. C., Dixit A. A., Fortmann S. P., Linde R. B. and Palaniappan, L. P., Type
2 diabetes: identifying high risk Asian American subgroups in a clinical Population, Diabetes Res. Clin. Pract. 93(2), 248-254 (2011).

[49] Choi S. E., Liu M., Palaniappan L. P, Wang E. J. and Wong N. D., Gender and ethnic differences in the prevalence of type 2 diabetes among Asian subgroups in California, J.Diabetes Complications. 27(5), 429-435 (2013).

[50] Hastings K. G., Jose P. O., Kapphahn K. I., Frank A. T. H., Goldstein B. A., ThompsonC. A. et al., Leading causes of death among Asian American subgroups (2003-2011),PLoS One, 10(4), e0124341 (2015).

[51] Hammoud T, Tanguay J. F. and Bourassa M. G., Management of coronary artery disease: therapeutic options in patients with diabetes, J.Am. Coll. Cardiol. 36, 355-65(2000).

[52] Naito R. and Miyauchi K., Coronary artery disease and type 2 diabetes mellitus: current treatment strategies and future perspectives, Int. Heart J. 58, 475-480 (2017).

[53] Chung J. O., Park S., Cho D. H., Chung D. J. and Chung M. Y., Anemia, bilirubin, and cardiovascular autonomic neuropathy in patients with type 2 diabetes, Medicine. 96,15(e6586) (2017)

[54] Yu Y., Ouyang X., Lou Q., Gu L., Mo Y., Ko G. T. et al., Validity of glycated hemoglobin in screening and diagnosing type 2 diabetes mellitus in Chinese subjects, Korean J. Int. Med. 27, 41-46 (2012).

[55] Araneta M. R., Grandinetti A. and Chang H.,A1C and diabetes diagnosis among Filipino Americans, Japanese Americans, and Native Hawaiians, Diabetes Care. 33,2626-2628, 14, 58-66 (2010).

Citation: Deovina N. Jordan, James L. Jordan, Neil John Yumul, Janine Marie Baptista. Errors Associated with Aggregating Asian American Ethnic Groups in Diabetes Research, Education, and Clinical Practice. ARC Journal of Diabetes and Endocrinology.2017; 3(2):1-10. doi:dx.doi.org/10.20431/2455-5983.0302001.

Copyright: (C) 2017 Authors. This is an open-access article distributed under the terms of the Creative Commons Attribution License, which permits unrestricted use, distribution, and reproduction in any medium, provided the original author and source are credited. 